Original Article

\title{
Deep learning for elective neck delineation: More consistent and time efficient
}

\author{
J. van der Veen ${ }^{\mathrm{a}, 1}$, S. Willems ${ }^{\mathrm{b}, 1}$, H. Bollen ${ }^{\mathrm{a}}$, F. Maes ${ }^{\mathrm{b}}$, S. Nuyts ${ }^{\mathrm{a}, *}$

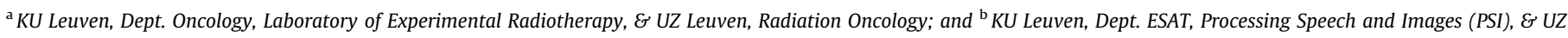 \\ Leuven, Medical Imaging Research Center, Leuven, Belgium
}

\section{A R T I C L E I N F O}

\section{Article history:}

Received 23 July 2020

Received in revised form 30 September

2020

Accepted 5 October 2020

Available online $\mathrm{xxxx}$

\section{Keywords:}

Head and neck neoplasms

Radiotherapy

Elective nodal target volumes

Observer variation

Neural networks (computer)

Delineation

\begin{abstract}
A B S T R A C T
Background/purpose: Delineation of the lymph node levels of the neck for irradiation of the elective clinical target volume in head and neck cancer (HNC) patients is time consuming and prone to interobserver variability (IOV), although international consensus guidelines exist. The aim of this study was to develop and validate a 3D convolutional neural network (CNN) for semi-automated delineation of all nodal neck levels, focussing on delineation accuracy, efficiency and consistency compared to manual delineation. Material/methods: The CNN was trained on a clinical dataset of $69 \mathrm{HNC}$ patients. For validation, 17 lymph node levels were manually delineated in 16 new patients by two observers, independently, using international consensus guidelines. Automated delineations were generated by applying the CNN and were subsequently corrected by both observers separately as needed for clinical acceptance. Both delineations were performed two weeks apart and blinded to each other. IOV was quantified using Dice similarity coefficient (DSC), mean surface distance (MSD) and Hausdorff distance (HD). To assess automated delineation accuracy, agreement between automated and corrected delineations were evaluated using the same measures. To assess efficiency, the time taken for manual and corrected delineations were compared. In a second step, only the clinically relevant neck levels were selected and delineated, once again manually and by applying and correcting the network.

Results: When all lymph node levels were delineated, time taken for correcting automated delineations compared to manual delineations was significantly shorter for both observers (mean: 35 vs 52 min, $\mathrm{p}<10^{-5}$ ). Based on DSC, automated delineation agreed best with corrected delineation for lymph node levels Ib, II-IVa, VIa, VIb, VIIa, VIIb (DSC >85\%). Manual corrections necessary for clinical acceptance were $1.4 \mathrm{~mm}$ MSD on average and were especially low $(<1 \mathrm{~mm})$ for levels II-IVa, VIa, VIIa and VIIb. IOV was significantly smaller with automated compared to manual delineations (MSD: $1.4 \mathrm{~mm} \mathrm{vs} 2.5 \mathrm{~mm}$, $\mathrm{p}<10^{-11}$ ). When delineating only the clinically relevant neck levels, the correction time was also significantly shorter (mean: 8 vs $15 \mathrm{~min}, \mathrm{p}<10^{-5}$ ). Based on DSC, automated delineation agreed very well with corrected delineation (DSC > 87\%). Manual corrections necessary for clinical acceptance were $1.3 \mathrm{~mm}$ MSD on average. IOV was significantly smaller with automated compared to manual delineations (MSD: $0.8 \mathrm{~mm}$ vs $2.3 \mathrm{~mm}$, p $<10^{-3}$ ).

Conclusion: The CNN developed for automated delineation of the elective lymph node levels in the neck in HNC was shown to be more efficient and consistent compared to manual delineation, which justifies its implementation in clinical practice.
\end{abstract}

(C) 2020 Elsevier B.V. All rights reserved. Radiotherapy and Oncology xxx (2020) xxx-xxx
Abbreviations: CNN, Convolutional neural network; CTV, Clinical target volume; DSC, Dice similarity coefficient; HD, Hausdorff distance; HNC, Head and neck cancer; MSD, Mean surface distance; Mm, Millimetres; IOV, Interobserver variability; IntraOV, Intraobserver variability; RT, Radiotherapy.

* Corresponding author at: KU Leuven, Dept. Oncology, Laboratory of Experimental Radiotherapy, \& UZ Leuven, Radiation Oncology, B-3000 Leuven, Belgium.

E-mail address: sandra.nuyts@uzleuven.be (S. Nuyts).

1 These authors contributed equally to this work.
Radiotherapy (RT) is one of the cornerstones in the treatment of head and neck cancer (HNC), either alone, after surgery or concomitantly with chemotherapy. Delineation of organs at risk (OARs) and clinical target volumes (CTV) is an important step in the treatment process and is necessary before the treatment plan can be created. This step is typically done manually and is a time-consuming activity. It requires intensive training before it can be done correctly and is susceptible to intra-observer (IntraOV) and interobserver variability (IOV), even when guidelines are avail- 
able [1-6]. Correct delineation is essential for accurate RT treatment planning and interpretation of dose volume histograms (DVH) to ensure the best RT quality. Adaptive RT (ART) is an approach to correct for changes in anatomy and tumour shape/volume during the RT course (online and offline) to ensure adequate tumour coverage while keeping dose to OARs as low as possible. This concept has been around for several years but technical limitations have prevented its implementation in routine clinical practice. Some patients may require multiple adaptations with repeated manual delineation of a new planning $\mathrm{CT}$, making the procedure tedious and prone to IOV. To use resources more effectively, these steps would ideally be replaced by automated processes with minimal input from clinicians.

Studies have been performed which showed the advantage of atlas-based automatic delineation of OARs and CTVs, but due to differences in patient anatomy, primary tumour sites and adenopathies, multiple atlases are needed [7-10]. Deep learning algorithms offer an elegant solution to this problem as they can be trained on a large and heterogeneous population dataset. They have already proven to be useful in automated delineation of OARs by significantly improving delineation time and consistency compared to manual delineations [11-14]. Automated delineation of high risk oropharyngeal CTV has also been investigated and the predicted contour provided close agreement to the ground-truth [15]. For nasopharyngeal tumours specifically, automated delineation of the primary gross tumour volume (GTV) and high risk and elective CTVs was acceptable according to Men et al. and showed potential to improve the consistency of contouring [16]. Wong et al. investigated the performance of automatic segmentation of neck levels Ib, II, III, IV, V for which the performance was not higher than the interobserver variability shown in the study [12]. These recent studies only evaluated the total elective clinical target volume (CTVetot) incorporating only neck levels relevant to that primary HNC location. However, this is not generalisable to other primary HNC locations and tumour stages which may require different neck levels to be segmented. Therefore we established a more general approach which can be used for all HNC patients unrelated to the HNC subtype and the specific neck levels required for radiotherapy treatment planning. To our knowledge, deep learning for automated delineation of all elective nodal volumes separately, unrelated to HNC locations has as of yet not been investigated.

In a previous publication we have shown that despite implementation of international consensus guidelines [17-20], there is significant IOV in both nodal level selection and delineation amongst HNC radiation oncologists [1]. This has led to the design of the current study, to develop and evaluate the potential of a 3D convolutional neural network ( $\mathrm{CNN})$ for automated delineation of all nodal neck levels, which could significantly reduce delineation time and IOV. This would not only be useful in the current RT setting resulting in better treatment standardisation and more efficient use of clinical time for routine contour generation, but also a first step towards enabling future offline ART workflows.

\section{Methods}

\section{$3 D$ convolutional neural network}

A 3D CNN, with an architecture based on the work from Kamnitsas et al. [21] and exactly the same as the architecture used for OAR segmentation in HNC patients as published in our previous work $[11,22]$, was reimplemented for automated delineation of the lymph node levels in the neck (see supplementary materials A for specific details). The CNN was trained from scratch on a random selection of 69 CT scans from previously treated HNC patients; 66 contrast-enhanced - 3 without contrast, 18 post-operative (anatomy still recognisably intact, so for example total laryngec- tomy excluded). This included different primary treating sites such as oropharynx, nasopharynx, hypopharynx, oral cavity, larynx and carcinomas of unknown primary. CT images were acquired in the supine position with custom thermoplastic mask for immobilization, according to the conventional clinical protocol. A multidetector-row spiral CT scanner was used (Somatom Sensation Open, 40-slice configuration; Siemens Medical Solutions, Erlangen, Germany). The acquisition parameters were: $120 \mathrm{kvP} / 230 \mathrm{mAs}$ (quality reference $\mathrm{mAs}$ with CARE Dose4D), no gantry tilt, spiral mode, rotation time $1 \mathrm{~s}, 40$ detector rows at $0.6 \mathrm{~mm}$ intervals, table speed $21.6 \mathrm{~mm} /$ rotation (pitch $=0.9$ ), reconstruction interval $3 \mathrm{~mm}$ using Kernel B30s medium smooth, matrix size $512 \times 512$, pixel spacing $0.97 \times 0.97 \mathrm{~mm}$. For the training set 21 lymph node levels were manually delineated in each patient by a single expert radiation oncologist using the international delineation consensus guidelines [17]. These levels were: central levels Ia, VIa and VIb and bilateral levels Ib, II, III, IVa, IVb, V, Vc, VIIa and VIIb. The bilateral levels were delineated at both sides of the neck separately. Because in clinical practice levels II, III and IVa are often selected as one target volume, they were grouped together as one level (II-IVa), which resulted in a total of 17 neck levels per patient. The CNN was trained to delineate each level separately such that when the network is implemented in clinical routine, each level can be individually selected by the treating radiation oncologist to include in the elective clinical target volume (CTVetot), i.e. the union of all relevant neck levels used for radiotherapy treatment planning.

\section{Data acquisition test set}

Patients were recruited between March and April 2020 and included consecutive patients with a newly diagnosed HNC, scheduled for RT at UZ Leuven. All tumour sites and disease stages were included, except patients who underwent a total laryngectomy because this distorted the anatomy too much and would also affect the manual delineation. In total, 16 patients were included in the study of which five were post-operative patients (Table 1). All patients had a planning CT scan (13 contrast-enhanced, 3 not contrast-enhanced) acquired with the same scanner and the same settings as described above. Note that the CNN was trained on CT scans from the same scanner and same institution as the scans for our clinical evaluation.

\section{Study design}

Per patient, a template was created including 16 OARs and the gross tumour volume (GTV) and clinical target volume (CTV) of the primary tumour and adenopathies. The following OARs were delineated with a clinically validated CNN for OAR delineation after which they were carefully corrected if needed: brainstem, spinal cord, parotids glands, submandibular glands, oral cavity, mandible, supraglottic larynx, glottic area, cochleas and pharyngeal constrictor muscles [11]. The CNN trained for lymph node level delineation was applied to all 16 patients, yielding original, unmodified automated delineations for each of the 17 neck levels. The study was split into two parts to evaluate the CNN performance and its clinical benefits.

In the first study part, all neck levels were evaluated separately. Two observers, trained in delineation of elective CTVs, manually delineated the 17 neck levels separately ("manual delineations") (Eclipse, Varian Medical Systems, Palo Alto, CA). In a separate, uninterrupted session, each case was delineated a second time by modifying and correcting the presented automated delineations generated by the CNN to create the "corrected delineations" (Fig. 1). In half of the cases the manual delineations were done first with corrections of the automated delineations minimum 2 weeks 
Table 1

Patient characteristics.

\begin{tabular}{|c|c|c|c|c|c|c|c|}
\hline $\mathrm{Nr}$ & Site & $\begin{array}{l}\text { TNM 8th } \\
\text { edition }\end{array}$ & $\begin{array}{l}\text { Primary RT or } \\
\text { postoperative }\end{array}$ & Contrast & Artefacts & Large errors in automated delineation and possible reasons & $\begin{array}{l}\text { Levels segmented in clinical } \\
\text { practice }\end{array}$ \\
\hline 1 & Oral cavity & rpT3NO & Postoperative & yes & $\begin{array}{l}\text { Minor scatter from clips and } \\
\text { osteosynthesis material }\end{array}$ & Level IbL and IIL due to clips and post-operative anatomy change & None \\
\hline 2 & CUP & cT0N3b & Primary & $\begin{array}{l}\text { No, kidney } \\
\text { failure }\end{array}$ & none & $\begin{array}{l}\text { Level IIR due to large adenopathy, level IV due to enlarged thyroid } \\
\text { gland. Exclusion of part of common carotid artery }\end{array}$ & $\begin{array}{l}\text { Right: IB, II-IVa, V, VIIa, VIIb } \\
\text { Left: none }\end{array}$ \\
\hline 3 & Oropharynx & cT1N3b & Primary & yes & Minor scatter from dental implants & Level IIL due to large adenopathy & $\begin{array}{l}\text { Right: II-Iva } \\
\text { Left: Ib, II-IVa, V, VIIa, VIIb }\end{array}$ \\
\hline 4 & Oral cavity & pT4aNO & Postoperative & yes & Minor scatter from dental implants & Level IbR and IIR due to clips and post-operative anatomy change & None \\
\hline 5 & Supraglottis & cT3NO & Primary & yes & none & Level VIa not visible due to blurry CT scan & Bilateral: II-IVa, Vla, VIb \\
\hline 6 & Oropharynx & cT3N2b & Primary & yes & none & - & $\begin{array}{l}\text { Right: II-Iva } \\
\text { Left: Ib, II-IVa, VIIa }\end{array}$ \\
\hline 7 & Supraglottis & cT2NO & Primary & yes & Minor scatter from dental implants & Level IV due to enlarged thyroid gland & Bilateral: II-IVa \\
\hline 8 & Oropharynx & cT2NO & Primary & yes & Minor scatter from dental implants & - & Bilateral: II-IVa, VIIa \\
\hline 9 & Supraglottis & cT3N2b & Primary & yes & Minor scatter from dental implants & - & $\begin{array}{l}\text { Right: II-IVa, Via } \\
\text { Left: Ib, II-IVa, V, VIIa }\end{array}$ \\
\hline 10 & Nasopharynx & cT1N3 & Induction chemo & yes & Major scatter from dental implants & - & $\begin{array}{l}\text { Bilateral: IB, III-IVa, IVb, V, VIIa, } \\
\text { VIIb }\end{array}$ \\
\hline 11 & Oropharynx & cT2NO & Primary & yes & none & - & Bilateral: II-IVa, VIIa \\
\hline 12 & Skin & $\mathrm{pN} 2 \mathrm{a}$ & Postoperative & $\begin{array}{l}\text { No, not } \\
\text { required }\end{array}$ & none & Level IbR due to clips and post-operative anatomy change & None \\
\hline 13 & CUP & cT0N3 & Primary & yes & Minor scatter from dental implants & Level II-IVaL due to large adenopathy & $\begin{array}{l}\text { Right: II-IVa, VIIa } \\
\text { Left: Ib, II-IVa, IVb, V, VIIa, VIIb }\end{array}$ \\
\hline 14 & Oropharynx & cT4N1 & Primary & $\begin{array}{l}\text { No, kidney } \\
\text { failure }\end{array}$ & none & $\begin{array}{l}\text { Level IIL due to adenopathy, exclusion of part of common carotid } \\
\text { artery }\end{array}$ & $\begin{array}{l}\text { Right: II-Iva } \\
\text { Left: Ib, II-IVa, V, VIIa, VIIb }\end{array}$ \\
\hline 15 & $\begin{array}{l}\text { Oral cavity nodal } \\
\text { recurrence }\end{array}$ & $(\mathrm{pT} 2) \mathrm{pN} 1$ & Postoperative & yes & $\begin{array}{l}\text { Minor scatter from dental implants and } \\
\text { clips }\end{array}$ & $\begin{array}{l}\text { Level II-IVa, V and Vc left due to clips and post-operative anatomy } \\
\text { change }\end{array}$ & $\begin{array}{l}\text { Right: Ib, II-Iva } \\
\text { Left: Ib, II-IVa, V }\end{array}$ \\
\hline 16 & Oral cavity & pT2N1 & Postoperative & yes & $\begin{array}{l}\text { Major scatter from dental implants and } \\
\text { clips }\end{array}$ & Cranial level Vla because of post-operative anatomy change & $\begin{array}{l}\text { Right: Ib, II-Iva } \\
\text { Left: none }\end{array}$ \\
\hline
\end{tabular}

Abbreviations: CUP: cancer of unknown primary; L: left; N: clinical nodal stage; R: right; TNM: tumour staging according to the TNM-8 staging system (2017); T: clinical tumour stage 


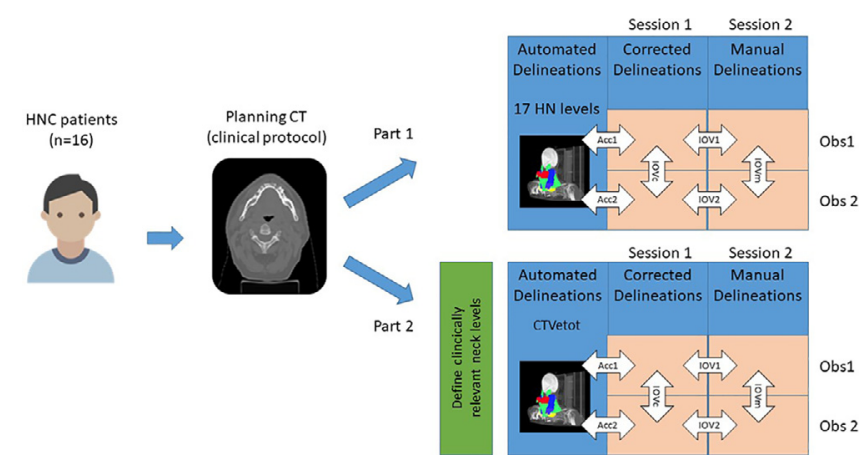

Fig. 1. Overview of study design. Automated delineations of 17 lymph node levels in the neck in conventional planning CT images of $16 \mathrm{HNC}$ patients were corrected by 2 different observers (obs 1 and obs 2 ) and were also manually delineated by the same observers in different delineation sessions (Part 1). Accuracy of automated delineation was assessed by comparing automated and corrected contours for each observer (Acc1 for observer 1, Acc2 for observer 2). Intra-observer variability was assessed by comparing corrected and manual delineations by the same observer (IntraOV1 and IntraOV2). Inter-observer variability was assessed by comparing corrected and manual delineations by different observers (IOVc and IOVm). In Part 2 , the clinically relevant neck levels were defined and only these levels were grouped together into CTVetot and corrected where necessary, and also delineated manually in one volume.

later, while in the other half the correction of the automated delineations was done first with manual delineation performed 2 weeks later. The observers were blinded for each other's delineations but also for their own delineations made in the first session. To prevent bias, an average interval of two weeks had to be respected between both delineation sessions by the same observer for the same patient.

In the second study part, the same observers had to manually segment only the relevant elective neck levels in one structure, i.e. CTVetot depending on tumour stage and location. All cases were delineated a second time by selecting the relevant neck levels from the automated delineations and merged into one structure which was corrected if necessary. To avoid IOV by selecting different neck levels, the required neck levels for each patient were known beforehand and variability between observers only involved variability in segmentation. Hence, IOV and intraOV due to differences in nodal selection was not evaluated in this study. To avoid bias, the second part of the study was performed two months later than the first part with again an average interval of 2 weeks between both delineation sessions.

The contours the two observers delineated (manual and corrections in part 1 and 2) were evaluated by a third observer (SN), who would correct them if necessary.

\section{Validation}

To validate the benefits of a CNN based automated delineation tool, accuracy, impact on IOV and IntraOV and time efficiency were assessed for all lymph node levels and the CTVetot.

\section{Efficiency}

Each observer recorded the time needed to complete the manual delineations and to correct the automated delineations for each patient. This included the time for adjusting window settings, navigating between slices and creating or correcting all delineations for all 17 lymph node levels and CTVetot.

\section{Performance}

The accuracy (Acc) of the automated delineation tool was assessed for each observer and each level separately by comparing it to the corrected delineations and the manual delineations using the Dice Similarity Coefficient (DSC) and the Mean Surface Distance (MSD). DSC is a measure for the overlap between two delineations $A$ and $B$, yielding a value of $100 \%$ in case of perfect overlap and a value of $0 \%$ if no overlap:

$\operatorname{DSC}(\%)=2 * \frac{|A \cap B|}{|A|+|B|} * 100 \%$

with $|A|$ and $|B|$ the volumes of each delineation and $|A \cap B|$ the volume of their intersection. MSD represents the mean distance between two delineations $A$ and $B$ in $\mathrm{mm}$ :

$\operatorname{MSD}(A, B)=\frac{h(A, B)+h(B, A)}{2}$

$h(A, B)=\operatorname{mean}_{a \in A}\left\{\min _{b \in B}\{d(a, b)\}\right\}$

with $d(a, b)$ the 3D distance between point $a$ on delineation $A$ and point $b$ on delineation $B$. Both DSC and MSD provide an indication for the amount of corrections necessary for clinical approval.

The Hausdorff-distance (HD) is calculated as the maximum distance between any two 2 closest points on both delineations in either way:

$H D(A, B)=\max \left\{\max _{a \in A}\left\{\min _{b \in B}\{d(a, b)\}\right\}, \max _{b \in B}\left\{\min _{a \in A}\{d(b, a)\}\right\}\right\}$

HD95 considers the 95th-percentile of the closest point distances instead of their maximum [23].

\section{Variability}

The impact of the automated delineation tool on IOV was assessed for each delineated level separately and the CTVetot by computing DSC, MSD and HD95 between the manual (IOVm) and between the corrected delineations (IOVc) made by both observers (with larger DSC, smaller MSD and smaller HD95 indicating less IOV). In addition, intra-observer variability for the same observer was assessed by comparing manual and corrected delineations made by the same RO.

\section{Statistical analysis}

Statistically significant differences for DSC, MSD, HD and volume were assessed with a two-sided, paired Wilcoxon signed rank test and delineation time reduction was assessed with a paired two-sample Student's t-Test, using significance level $\alpha=0.05$ and a power of $90 \%$.

\section{Results}

In part 1 , there were on average 14.8 days between the manual and corrected delineation sessions with a minimum of 13 days and a maximum of 20 days. The computation time needed by the CNN to automatically delineate all 17 levels was on average 68 seconds per patient. Fig. 2 shows the time required for both manual delineations and correcting the automatic delineation for all neck levels separately and CTVetot. The time needed to review and correct the automated delineations of all neck levels was for both observers significantly shorter than the time required for manual delineation of all neck levels (Observer 1: 29 vs 49 minutes ( $<<10^{-5}$ ); Observer 2: 41 vs $54 \mathrm{~min}\left(\mathrm{p}<10^{-5}\right)$; mean: 35 vs $\left.52 \mathrm{~min}, \mathrm{p}<10^{-5}\right)$. For part 2 , there were on average 12.2 days between the manual and corrected delineation sessions with a minimum of 12 days and a maximum of 15 days. The time necessary to select the clinically relevant levels from the CNN predictions and merge the contours into one CTVetot took on average $23 \mathrm{~s}$. The time necessary to 

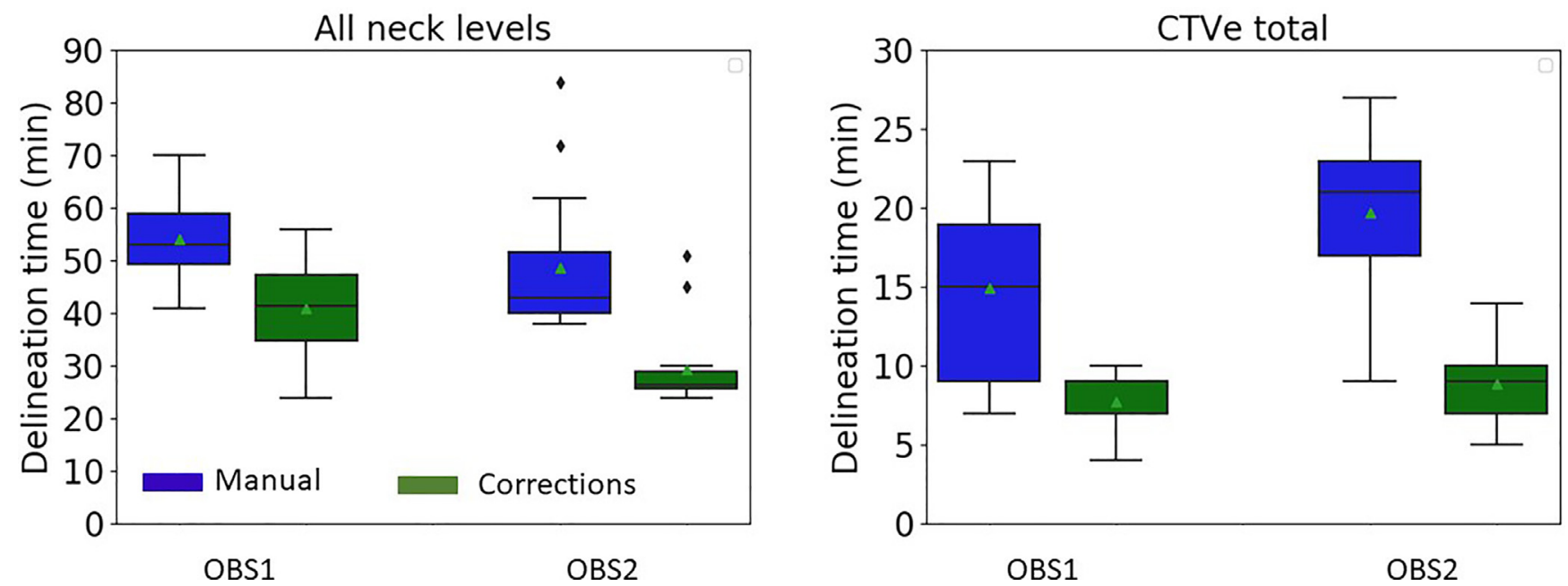

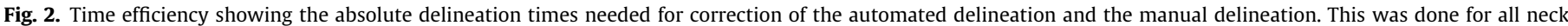

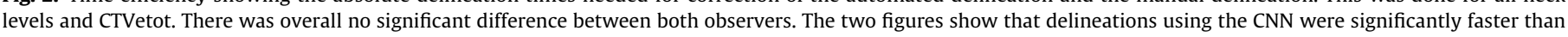
manual delineations for both observers (obs 1 and obs 2).

review the merged CTVetot contour was also significantly shorter for both observers than performing manual delineation of CTVetot (Observer 1: 7 vs 15 min $\left(\mathrm{p}<10^{-5}\right)$; Observer 2: 9 vs $15 \mathrm{~min}$ $\left(\mathrm{p}<10^{-5}\right)$; mean: 8 vs $\left.15 \mathrm{~min},\left(\mathrm{p}<10^{-5}\right)\right)$.

The third observer (SN), made no changes to the delineations observers 1 and 2 made, as they were acceptable for clinical use.

To investigate the performance of the automated delineations, mean DSC, MSD and HD95 for automated versus corrected delineations and for automated versus manual delineations were calculated and are summarised in Table 2. The average volume of the manual delineations of the two observers is added in a last column. In general, all validation metrics are significantly better when comparing the automated delineations with the corrected delineations since only parts are adapted which are clinically relevant and automatically lead to more conformity. Hence these values give an upper limit of the performance while the metrics comparing the automated contours with the manual contours rather provide the lower limit performance.

Based on DSC, the network performed best for CTVetot and lymph node levels Ib, II-IVa, VIa, VIb, VIIa, VIIb (DSC > 85\%). The average MSD was $\leq 1 \mathrm{~mm}$ for levels Ia, II-IVa, VIa, VIIa and VIIb. Average MSD for all other neck levels and CTVetot were below $3 \mathrm{~mm}$ with HD95 laying between 2.0 and $14.0 \mathrm{~mm}$. For most levels, there was no significant difference in corrections needed by both observers for clinical acceptance of automated delineations. Examples of manual, automated and corrected delineations are shown in Fig. 3.

Fig. 4 shows a significant reduction in IOV in terms of MSD, DSC and HD95 with corrected compared to manual delineations for most neck levels and CTVetot. IOVc was smaller than intraobserver variability for most elective neck levels. IOV was significantly smaller with automated compared to manual delineations (MSD: $0.8 \mathrm{~mm}$ vs $2.3 \mathrm{~mm}, \mathrm{p}<10^{-3}$, DCS: $92.2 \%$ vs $79.8 \%$, $\mathrm{p}<10^{-3}$ and HD95: $4.1 \mathrm{~mm}$ vs $8.6 \mathrm{~mm}, \mathrm{p}<10^{-3}$ ).

\section{Discussion}

The aim of this study was to evaluate the benefits of an automated delineation tool for delineation of elective lymph node levels in the neck, with regard to delineation accuracy, efficiency and impact on IOV. Automated delineation was performed by a $\mathrm{CNN}$, which was trained using manual delineation of 21 separate lymph node levels in the neck of HNC patients in agreement with international consensus guidelines [17]. To our knowledge, no other studies have trained a CNN to delineate all elective neck levels separately, so that the treating radiation oncologist can select the relevant elective neck levels subsequently. Therefore, we trained a CNN to segment all neck levels and tested its efficiency and accuracy in part 1 . In part 2 we tested how this would work in a clinical setting by only selecting the clinically relevant levels and investigated the impact on IOV.

Improved delineation efficiency with the CNN was demonstrated by a reduction in time needed to review and correct the automated delineations of all neck levels versus the time needed for manual delineations, which improved significantly for both observers (part 1). This was also the case for part 2 of the study, where we were able to show that the time taken for selecting the necessary neck levels and correcting them where necessary, was significantly shorter than delineating CTVetot manually (average of 8 vs 15 min, $\mathrm{p}<10^{-5}$ ). In general, delineation of all levels separately (part 1 ) required more time compared to only segmenting the relevant neck levels in one single volume (part 2, CTVetot), because less levels were segmented and the boundaries between different levels did not need to be taken into account, resulting in shorter delineation times. Although correction of the automated delineations was usually shorter than the manual delineations, automated delineations required on average more corrections for post-operative patients, patients with adenopathies or patients who received a non-contrast-enhanced CT scan. Additionally, the observers were used to making manual delineations but had to learn to correct the contours generated by the $\mathrm{CNN}$, which resulted in a learning curve, and shorter correction time towards the end of the study. Overall, correcting automated contours leads to an improved efficiency in clinical practice compared to manual delineations, for all neck levels separately as well as for a merged contour containing only the relevant neck levels. With an average time of $8 \mathrm{~min}$ to correct CTVetot, this auto-delineation tool can be used for segmentations in routine clinical practice and for offline ART, but still requires too much time to be used for online ART.

The performance of the CNN was evaluated in two ways. First by comparing the automated delineations to the manual delineations. This analysis showed a realistic lower limit of the performance with DSC ranging from $46 \%$ (level Vc) to $82 \%$ (level II-IVa). Wong et al. [12] reported a DSC of $72 \%$ for the total elective clinical target volume, which is comparable to our result, i.e. CTVetot of $76,7 \%$ for observer 1 and $73,5 \%$ for observer 2 . The advantage of 
Table 2

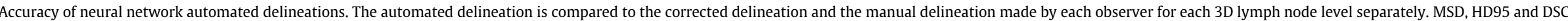

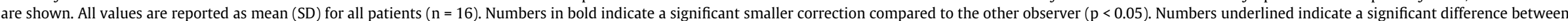
accuracy calculated using corrected contours and manual contours $(p<0.05)$. The last column provides average absolute volumes of the manually contoured neck levels in ml.

\begin{tabular}{|c|c|c|c|c|c|c|c|c|c|c|c|c|c|}
\hline & \multicolumn{6}{|c|}{ Corrected contours } & \multicolumn{6}{|c|}{ Manual contours } & \multirow{3}{*}{$\begin{array}{l}\text { AverageAbsolute Volume } \\
(\mathrm{ml})\end{array}$} \\
\hline & \multicolumn{3}{|c|}{ Observer 1 (Acc1) } & \multicolumn{3}{|c|}{ Observer 2 (Acc2) } & \multicolumn{3}{|c|}{ Observer 1 (Acc1m) } & \multicolumn{3}{|c|}{ Observer 2 (Acc2m) } & \\
\hline & $\operatorname{MSD}(\mathrm{mm})$ & HD95(mm) & DSC(\%) & $\operatorname{MSD}(\mathrm{mm})$ & HD95(mm) & DSC(\%) & $\mathrm{MSD}(\mathrm{mm})$ & HD95(mm) & $\operatorname{DSC}(\%)$ & $\mathrm{MSD}(\mathrm{mm})$ & HD95(mm) & DSC(\%) & \\
\hline LN_Ia & $1.0(1.9)$ & $3.4(5.5)$ & $\underline{84.5(31.8)}$ & $1.0(1.7)$ & $3.4(4.1)$ & $82.9(30.0)$ & $2.5(2.6)$ & $4.7(3.6)$ & $66.6(26.5)$ & $2.3(1.2)$ & $5.2(3.4)$ & $64.8(24.1)$ & $1.6(0.7)$ \\
\hline LN_IbL & $\underline{1.0(0.7)}$ & $\underline{5.0(3.7)}$ & $90.7(9.0)$ & $1.2(0.6)$ & $\underline{6.6(4.1)}$ & $\underline{88.1(8.0)}$ & $2.2(0.9)$ & $5.8(4.8)$ & $81.3(12.1)$ & $2.6(0.7)$ & $7.6(2.7)$ & $75.2(10.0)$ & 23.3(9.1) \\
\hline LN_IbR & $1.0(0.8)$ & $\underline{5.1(4.3)}$ & $89.5(9.1)$ & $1.3(1.0)$ & $\overline{7.8(5.9)}$ & $86.5(10.3)$ & $2.2(0.8)$ & $6.2(4.2)$ & 79.7(11.4) & $2.8(0.9)$ & $8.7(4.2)$ & $70.6(14.0)$ & $22.8(9.2)$ \\
\hline LN_234aL & $\underline{1.0(0.9)}$ & $\underline{4.7(3.3)}$ & $\underline{90.2(6.3)}$ & $\underline{0.9(0.7)}$ & $4.6(2.4)$ & $90.5(4.7)$ & $2.0(0.8)$ & $4.8(2.6)$ & $82.0(5.8)$ & $2.4(0.7)$ & $6.7(2.8)$ & $77.4(4.8)$ & $80.5(32.2)$ \\
\hline LN_234aR & $\underline{0.8(0.6)}$ & $\underline{4.5(4.1)}$ & $91.0(7.1)$ & $\underline{0.9(0.8)}$ & $5.5(5.0)$ & $90.4(8.5)$ & $1.8(0.6)$ & $5.6(4.3)$ & $82.0(7.8)$ & $2.5(0.7)$ & $7.8(4.6)$ & 75.3(9.1) & $73.9(21.2)$ \\
\hline LN_IVbL & $1.9(0.9)$ & $7.4(3.9)$ & $79.8(11.9)$ & $2.1(0.9)$ & $7.7(5.1)$ & $78.7(9.2)$ & $2.9(0.9)$ & $8.2(4.0)$ & 71.1(10.7) & $3.2(0.9)$ & $10.1(4.6)$ & $68.2(9.6)$ & $15.2(4.6)$ \\
\hline LN_IVbR & $\underline{2.0(1.0)}$ & $8.0(4.9)$ & $76.4(11.0)$ & $\underline{2.3(1.2)}$ & $8.5(4.9)$ & $75.1(13.9)$ & $3.0(1.0)$ & 7.3(3.6) & 69.5(10.5) & $3.7(1.2)$ & $11.3(8.0)$ & $62.3(9.4)$ & $16.1(3.8)$ \\
\hline LN_VL & $\underline{2.2(1.5)}$ & $12.3(11.1)$ & $78.3(13.8)$ & $1.9(1.5)$ & $14.0(11.9)$ & $77.7(17.4)$ & $3.4(1.9)$ & $13.6(10.7)$ & $63.8(12.6)$ & $3.8(1.9)$ & $16.4(12.9)$ & $60.3(13.5)$ & $17.9(8.6)$ \\
\hline LN_VR & $\underline{1.6(0.8)}$ & $9.5(5.9)$ & $81.9(8.6)$ & $\overline{1.1(0.7)}$ & $\overline{8.0(4.9)}$ & $\overline{85.4(10.3)}$ & $2.9(1.1)$ & $11.1(8.1)$ & $68.6(11.4)$ & $3.4(1.0)$ & $12.3(6.1)$ & $62.1(11.1)$ & $18.1(8.0)$ \\
\hline LN_VcL & $\underline{2.6(1.0)}$ & $7.8(3.7)$ & 71.3(12.4) & $2.6(1.3)$ & $9.6(5.2)$ & $66.1(18.3)$ & $3.9(1.2)$ & $10.0(5.2)$ & $57.3(11.0)$ & $4.3(1.0)$ & $12.1(4.7)$ & $49.8(15.9)$ & $13.7(8.0)$ \\
\hline LN_VcR & $\underline{2.3(0.8)}$ & 7.2(4.4) & 74.5(12.7) & $\underline{2.2(1.3)}$ & $\underline{8.9(6.9)}$ & $70.4(16.7)$ & $3.7(1.8)$ & $10.7(7.2)$ & $60.4(17.8)$ & $4.5(1.4)$ & $12.8(7.0)$ & $46.0(13.6)$ & $12.2(7.6)$ \\
\hline LN_VIa & $\underline{0.6(0.5)}$ & $\underline{4.3(3.5)}$ & $\underline{88.8(10.4)}$ & $\underline{0.8(0.7)}$ & $\underline{6.2(4.7)}$ & $\underline{87.1(10.7)}$ & $1.7(0.4)$ & $5.2(3.2)$ & $73.4(10.5)$ & $2.1(0.6)$ & $5.8(4.3)$ & $68.0(8.4)$ & $27.3(7.6)$ \\
\hline LN_VIb & $1.4(2.5)$ & $\overline{6.7(9.7)}$ & $\overline{87.8(16.0)}$ & $1.4(2.2)$ & $7.3(9.1)$ & $85.5(14.4)$ & $2.7(2.5)$ & $9.0(9.6)$ & 71.9(14.4) & $2.8(1.9)$ & $8.8(7.9)$ & $66.7(12.5)$ & $21.8(8.3)$ \\
\hline LN_VIIaL & $\underline{0.9(1.8)}$ & $5.3(8.3)$ & $\underline{85.0(19.7)}$ & $1.5(2.9)$ & 7.8(12.3) & $\underline{77.0(19.5)}$ & $2.0(1.4)$ & $7.5(7.5)$ & $59.8(15.0)$ & $2.5(2.3)$ & $9.6(11.5)$ & $47.7(14.9)$ & $1.9(0.5)$ \\
\hline LN_VIIaR & $0.4(0.3)$ & $2.0(2.0)$ & $90.0(8.7)$ & $0.7(0.4)$ & $\underline{5.1(4.4)}$ & $83.4(8.1)$ & $1.6(0.4)$ & $4.3(2.6)$ & $64.1(8.2)$ & $2.0(0.6)$ & $6.5(4.1)$ & $50.6(12.1)$ & $2.0(0.5)$ \\
\hline LN_VIIbL & $\underline{0.7(0.4)}$ & $\underline{3.1(1.8)}$ & $86.9(7.9)$ & $\overline{1.0(0.8)}$ & $4.8(5.1)$ & $81.9(14.6)$ & $1.6(0.5)$ & $4.0(2.0)$ & 73.9(11.3) & $2.1(0.6)$ & $4.9(2.0)$ & $66.4(14.3)$ & $4.5(1.1)$ \\
\hline LN_VIIbR & $\underline{0.5(0.4)}$ & $\underline{2.5(1.6)}$ & $\underline{89.7(7.5)}$ & $\underline{0.6(0.4)}$ & $3.5(2.4)$ & $89.2(7.3)$ & $1.5(0.3)$ & 3.4(1.3) & 78.4(7.3) & $2.1(1.0)$ & $5.3(3.5)$ & $69.2(16.7)$ & $5.2(1.2)$ \\
\hline CTVe_total & $\underline{1.2(0.7)}$ & $\underline{5.5(5.2)}$ & $\underline{89.5(7.8)}$ & $\underline{1.3(0.7)}$ & 7.4(7.2) & $\underline{87.8(7.5)}$ & $2.4(0.8)$ & $9.1(5.2)$ & $76.7(8.3)$ & $2.6(0.8)$ & $9.7(7.5)$ & $73.5(8.8)$ & $196.6(75.0)$ \\
\hline
\end{tabular}

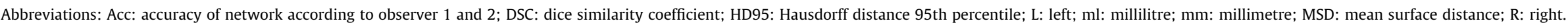




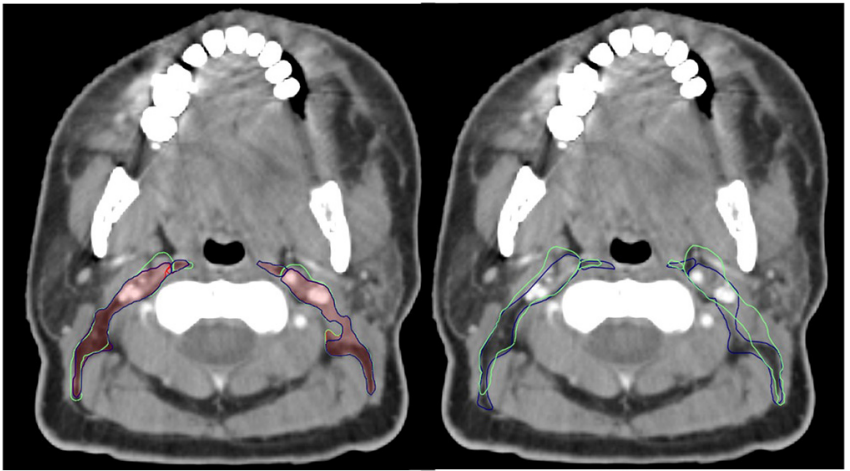

Fig. 3. Example of the difference between interobserver variability with manual (IOVm-right) vs corrected (IOVc-left) delineations. The red contours are the contours generated by the $\mathrm{CNN}$, with in the same image the corresponding corrected contours by the two observers. (For interpretation of the references to colour in this figure legend, the reader is referred to the web version of this article.)

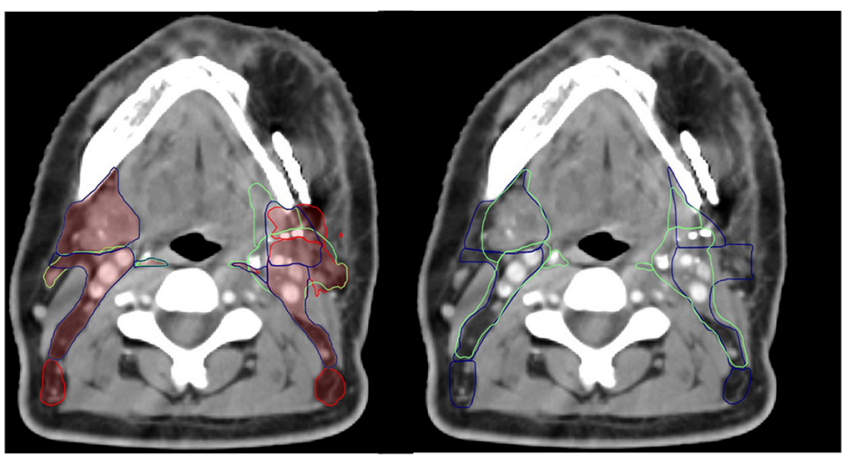

Fig. 3 (continued)

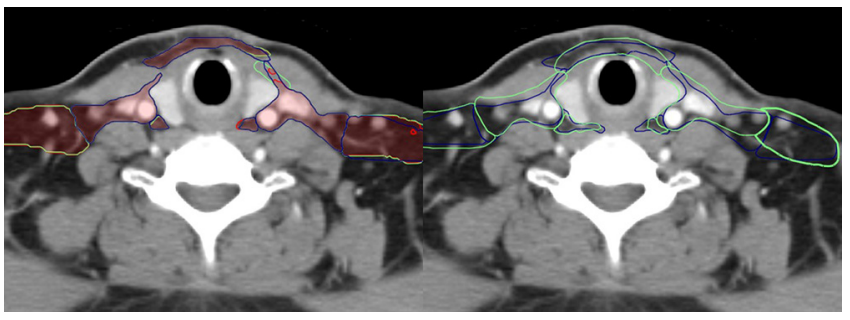

Fig. 3 (continued)

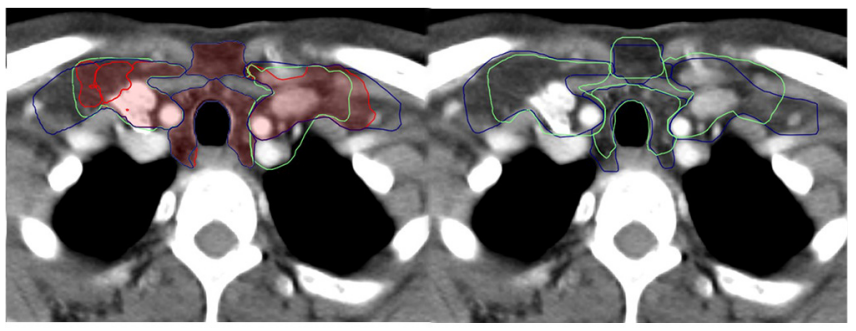

Fig. 3 (continued)

our network however is that it can be applied to all HNC patients because the neck levels can be selected separately. When comparing the results from table 2 (manual contours columns) to the data in Fig. 4, we can conclude that the accuracy of the generated contours compared to the manual contours, is within the range of the

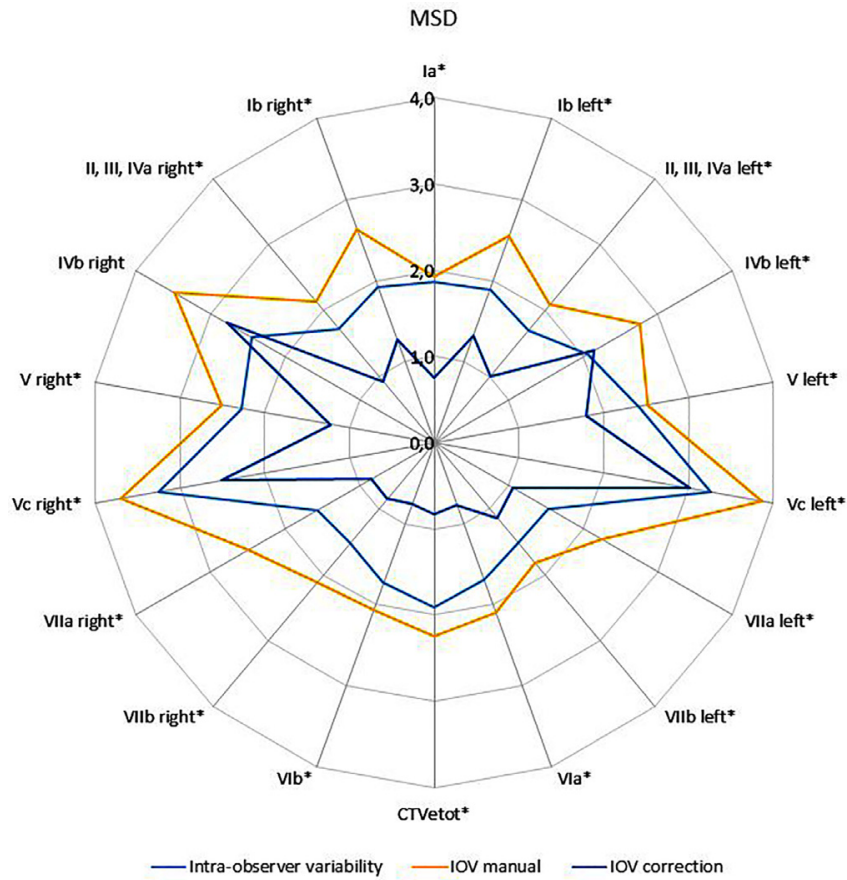

Fig. 4. Effect of the neural network on interobserver variability. IOVc was significantly smaller than IOVm for (a) MSD, (b) DSC and (c) HD95. This was the case for most of the elective neck levels, which are indicated with *. CTVetot also showed less IOV with the CNN compared to with manual delineation. Regarding MSD and DSC the IOV was less than intraobserver variability. Abbreviations: DSC: dice similarity coefficient; HD95: Hausdorff distance 95th percentile; IOVc: interobserver variability obtained by correcting automated delineations; IOVm: interobserver variability obtained with manual delineations; mm: millimetre; MSD: mean surface distance.

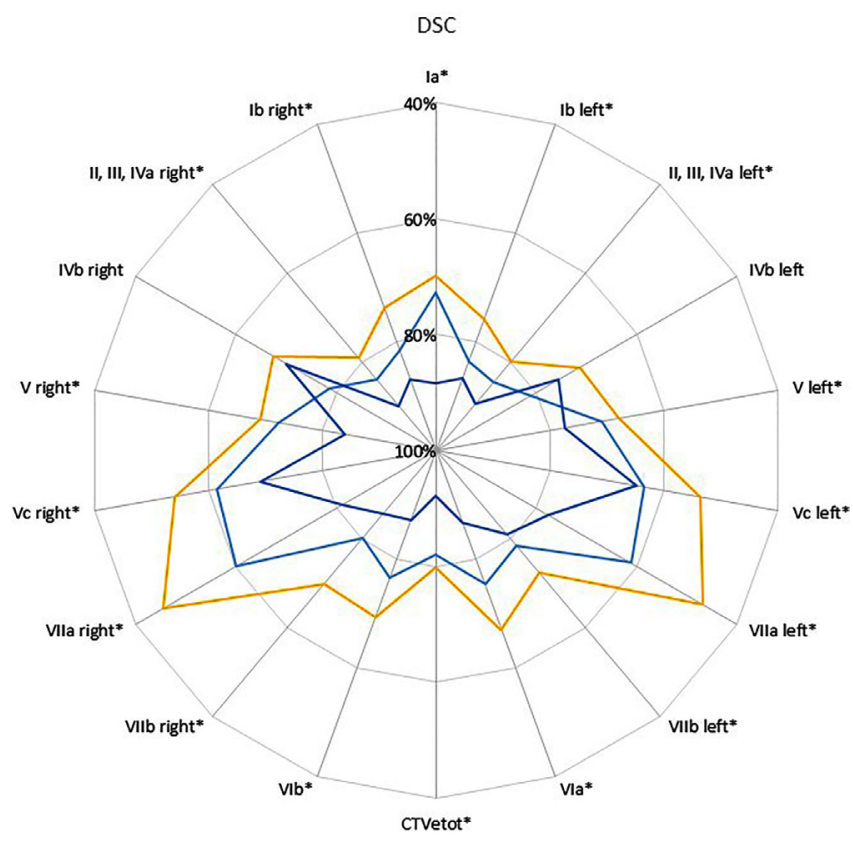

—Intra-observer variability - $10 \mathrm{~V}$ manual —IOV correction

Fig. 4 (continued)

IOV. A second comparison was done by assessing the necessary corrections made to the automated delineations to be clinically useful. This second analysis provided a realistic upper limit of the performance of the CNN since contours will be more conform 


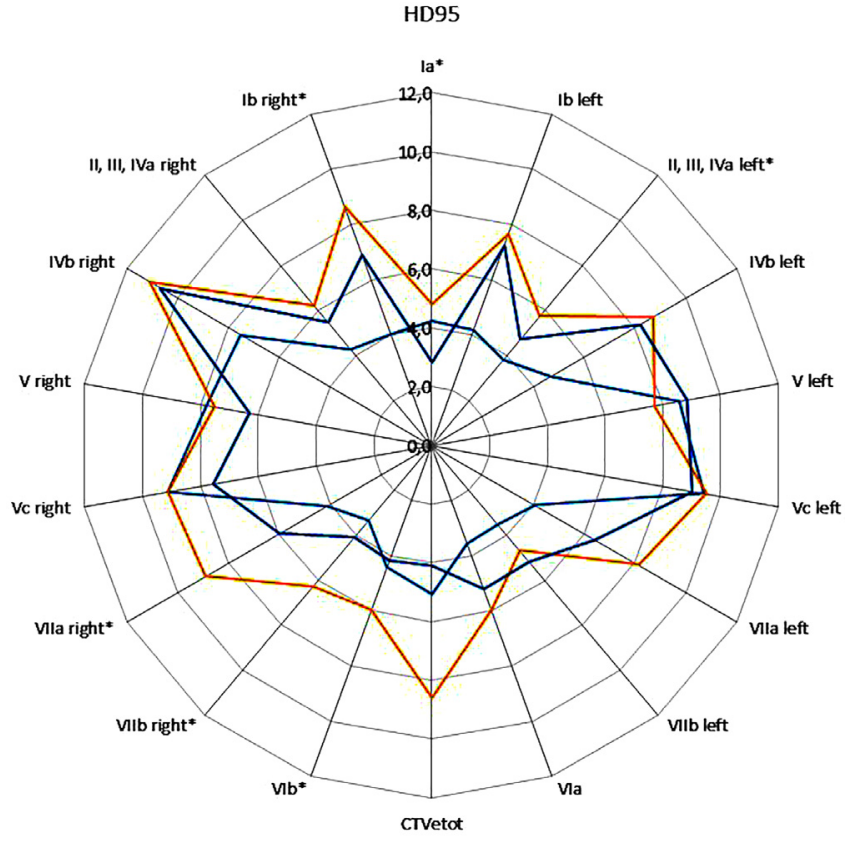

—Intra-observer variability - IOV manual - IOV correction

Fig. 4 (continued)

in this approach and therefore DSC ranged from 70,4\% (level Vc) to 91.0\% (level II-IVa). Following this delineation approach, corrections made by observer 1 were smaller than those made by observer 2 . Observer 1 was also the one who provided the manual delineations for the training set, hence this observer agreed slightly more with the CNN. As stated above, more corrections were needed for post-operative patients and patients with adenopathies or tumours bulging into the elective neck volumes due to a distorted anatomy or when patients had a CT scan without contrast enhancement (supplementary materials B). Another hurdle is patient positioning on the planning $\mathrm{CT}$, especially the degree of flexion or extension of the neck. If this differs too much from the patients used in the training set, this may cause the CNN to have difficulty in defining the borders of the neck levels.

The IOV, quantified by DSC, MSD and HD95 reduced significantly for most lymph node levels by using automated delineations compared to manual delineations. MSD decreased significantly for all lymph node levels, except right level IVb, and DSC improved significantly for all levels except bilateral level IVb. A reason for this exception could be that the lateral edge of the scalene muscle functions as lateral border for level IVb, and is often not clearly visible, resulting in more IOV. Regarding CTVetot, the IOV expressed in DSC improved from $79,8 \%$ (manual IOV) to $92,2 \%$ (corrected IOV). This manual IOV observed in our institution is comparable with the results shown by Wong et al, i.e. 79\% [12]. To conclude this part on IOV, we noticed that a contrast-enhanced planning CT makes contouring easier (both manual and automated), but that IOV still improved significantly in the automated delineation tract in the absence of contrast-enhancement. However, the combination of adenopathies in the neck and a none-contrast-enhanced planning CT do make it more difficult.

To our knowledge, this is the first study to investigate the feasibility of automated delineation of all clinically relevant elective nodal volumes in HNC planning CT scans using a CNN which is generalisable to all HNC patients. However, its benefits have already been shown in OAR delineation [11-14] delineation of high risk CTV [15] and some elective volumes in nasopharyngeal tumour irradiation [16]. When we compare the corrections made to the automated delineations for the lymph node levels defined in terms of DSC and MSD, these were larger than the corrections needed for OAR segmentation, which we assessed in an earlier published study [11]. This can be explained by the presence of adenopathies, which can alter the shape of the lymph node level when they are bulky, compared to the more general and consistent shapes of OARs over different subjects.

In another study, we assessed IOV between experts, in light of the international consensus guidelines (1). To be able to compare the results of both studies, the same neck levels selected in the prior study were selected in the current study to calculate DSC and MSD. When we compare IOV between experts with IOVm from the current study, these results are similar (DSC 0.77 and HD50 $1.9 \mathrm{~mm}$ vs DSC 0.72 and MSD $2.2 \mathrm{~mm}$ ), whereas the IOVc is smaller (DSC 0.87 and MSD $1.1 \mathrm{~mm}$ ).

The main strength of our study is the use of international consensus guidelines [17] to train the CNN to delineate the largest number of separate lymph node levels to date. The fact that the neck levels can be selected separately is of great added value because it allows the treating radiation oncologist to select the neck levels that should be irradiated depending on the risk of metastases. Therefore, in case guidelines for level selection were to be modified, as they recently were [24], the CNN could still be used. Even if the idea of elective nodal irradiation were to be replaced by sentinel guided neck irradiation [25,26] this CNN would still be appropriate, and if the guidelines for delineation were to be adjusted in the future, this CNN could easily be retrained.

A first limitation of this study is the use of single centre and single scanner data. Extra external validation is required to verify the generalisability to other centres. A possible second limitation is that the 69 patients used in the training set were delineated by only one observer (observer 1 ), which could have biased the network. However, guidelines were followed and the example delineations available in the supplementary data of the consensus guidelines [17] were used and feedback from an expert (SN) was given on the submitted delineations. As a result, the use of the automated delineation tool significantly reduced delineation time for both observers and significantly reduced IOV. On top of that, for most neck levels there was no significant difference in corrections between both observers (Ia, right Ib, II, III, Iva, IVb, left V, Vc, VIIb). The difference in time between manual and corrected delineations also did not differ significantly between both observers. A third possible limitation is that only two observers submitted contours in this study. Stronger conclusions could be drawn if using more observers. However manual IOV will always be influenced by the experience of additional observers (junior versus senior).

To conclude, we validated a CNN trained for automated delineation of lymph node levels in the neck in HNC patients based on international consensus guidelines in a clinical setting and showed that automated delineation is significantly more efficient ( 8 vs $15 \mathrm{~min}$ ) and reduces interobserver variability compared to manual delineations. Therefore, this auto-delineation tool can be used for segmentations in routine clinical practice and for offline ART, but still requires too much time to be used for online ART.

\section{Conflicts of interest}

None.

\section{Acknowledgments}

Siri Willems is supported by a Ph.D. fellowship of the research foundation - Flanders (FWO). Frederik Maes is supported by Inter- 
nal Funds KU Leuven under grant number C24/18/047. This research received funding from the Flemish Government (AI Research Program).

\section{Appendix A. Supplementary data}

Supplementary data to this article can be found online at https://doi.org/10.1016/j.radonc.2020.10.007.

\section{References}

[1] van der Veen J, Gulyban A, Nuyts S. Interobserver variability in delineation of target volumes in head and neck cancer. Radiother Oncol 2019;137:9-15.

[2] Brouwer CL, Steenbakkers RJ, van den Heuvel E, Duppen JC, Navran A, Bijl HP, et al. 3D variation in delineation of head and neck organs at risk. Radiat Oncol $2012 ; 7: 32$

[3] Geets X, Daisne J-F, Arcangeli S, Coche E, De Poel M, Duprez T, et al. Interobserver variability in the delineation of pharyngo-laryngeal tumor, parotid glands and cervical spinal cord: Comparison between CT-scan and MRI. Radiother Oncol 2005;77(1):25-31.

[4] Piotrowski T, Gintowt K, Jodda A, Ryczkowski A, Bandyk W, Bk B, et al. Impact of the intra- and inter-observer variability in the delineation of parotid glands on the dose calculation during head and neck helical tomotherapy. Technol Cancer Res Treat. 2014;tcrtexpress.201.

[5] Mukesh M, Benson R, Jena R, Hoole A, Roques T, Scrase C, et al. Interobserver variation in clinical target volume and organs at risk segmentation in postparotidectomy radiotherapy: can segmentation protocols help? $\mathrm{Br}$ J Radiol. 2012;85:e530-6.

[6] Peng YL, Chen L, Shen GZhu, Li YNing, Yao JJ, Xiao WWei, et al. Interobserver variations in the delineation of target volumes and organs at risk and their impact on dose distribution in intensity-modulated radiation therapy for nasopharyngeal carcinoma. Oral Oncol 2018;82:1-7.

[7] Daisne JF, Blumhofer A. Atlas-based automatic segmentation of head and neck organs at risk and nodal target volumes: A clinical validation. Radiat Oncol 2013;8:1-11.

[8] Commowick O, Grégoire V, Malandain G. Atlas-based delineation of lymph node levels in head and neck computed tomography images. Radiother Oncol 2008;87:281-9.

[9] Strassmann G, Abdellaoui S, Richter D, Bekkaoui F, Haderlein M, Fokas E, et al. Atlas-based semiautomatic target volume definition (CTV) for head-and-neck tumors. Int J Radiat Oncol, 2010;78:1270-6.

[10] Teguh DN, Levendag PC, Voet PWJ, Al-Mamgani A, Han X, Wolf TK, et al Clinical validation of atlas-based auto-segmentation of multiple target volumes and normal tissue (swallowing/mastication) structures in the head and neck. Int J Radiat Oncol Biol Phys 2011;81:950-7.

[11] van der Veen J, Willems S, Deschuymer S, Robben D, Crijns W, Maes F, et al. Benefits of deep learning for delineation of organs at risk in head and neck cancer. Radiother Oncol, 2019;138:68-74.
[12] Wong J, Fong A, McVicar N, Smith S, Giambattista J, Wells D, et al. Comparing deep learning-based auto-segmentation of organs at risk and clinical target volumes to expert inter-observer variability in radiotherapy planning. Radiother Oncol 2020;144:152-8.

[13] van Dijk LV, Van den Bosch L, Aljabar P, Peressutti D, Both S, Steenbakkers Roel JHM, et al. Improving automatic delineation for head and neck organs at risk by Deep Learning Contouring. Radiother Oncol 2020;142:115-23.

[14] Ibragimov B, Xing L. Segmentation of organs-at-risks in head and neck CT images using convolutional neural networks. Med Phys 2017;44:547-57.

[15] Cardenas CE, McCarroll RE, Court LE, Elgohari BA, Elhalawani H, Fuller CD, et al. Deep learning algorithm for auto-delineation of high-risk oropharyngeal clinical target volumes with built-in dice similarity coefficient parameter optimization function. Int J Radiat Oncol Biol Phys 2018;101:468-78.

[16] Men K, Chen X, Zhang Y, Zhang T, Dai J, Yi J, et al. Deep deconvolutional neural network for target segmentation of nasopharyngeal cancer in planning computed tomography images. Front Oncol. 2017;7:1-9.

[17] Grégoire V, Ang K, Budach W, Grau C, Hamoir M, Langendijk JA, et al. Delineation of the neck node levels for head and neck tumors: A 2013 update. DAHANCA, EORTC, HKNPCSG, NCIC CTG, NCRI, RTOG, TROG consensus guidelines. Radiother Oncol. 2014;110:172-81.

[18] Grégoire V, Levendag P, Ang KK, Bernier J, Braaksma M, Budach V, et al. CTbased delineation of lymph node levels and related CTVs in the node-negative neck: DAHANCA, EORTC, GORTEC, NCIC, RTOG consensus guidelines. Radiother Oncol 2003;69:227-36.

[19] Grégoire V, Eisbruch A, Hamoir M, Levendag P. Proposal for the delineation of the nodal CTV in the node-positive and the post-operative neck. Radiother Oncol. 2006;79(1):15-20.

[20] Grégoire V, Coche E, Cosnard G, Hamoir M, Reychler H. Selection and delineation of lymph node target volumes in head and neck conformal radiotherapy. Proposal for standardizing terminology and procedure based on the surgical experience. Radiother Oncol. 2000;56:135-50.

[21] Kamnitsas K, Ledig C, Newcombe VFJ, Simpson JP, Kane AD, Menon DK, et al. Efficient multi-scale 3D CNN with fully connected CRF for accurate brain lesion segmentation. Med Image Anal. 2017;36:61-78.

[22] Willems S, Crijns W, La Greca Saint-Esteven A, Van Der Veen J, Robben D, Depuydt T, et al. Clinical implementation of DeepVoxNet for auto-delineation of organs at risk in head and neck cancer patients in radiotherapy. In 2018:223-32.

[23] Grand Challenge: StructSeg, Internet Available from: https:// structseg2019.grand-challenge.org/Evaluation/ 2019.

[24] Biau J, Lapeyre M, Troussier I, Budach W, Giralt J, Grau C, et al. Selection of lymph node target volumes for definitive head and neck radiation therapy: a 2019 Update. Radiother Oncol 2019;134:1-9.

[25] Longton E, Lawson G, Bihin B, Mathieu I, Hanin F, Deheneffe S, et al. Individualized prophylactic neck irradiation in cNO head and neck cancer patients based on sentinel lymph node(s) identification: definitive results of a prospective phase I-II study. Int J Radiat Oncol. 2020.

[26] de Veij Mestdagh PD, Walraven I, Vogel WV, Schreuder WH, van Werkhoven E, Carbaat C, et al. SPECT/CT-guided elective nodal irradiation for head and neck cancer is oncologically safe and less toxic: A potentially practice-changing approach. Radiother Oncol 2020;147:56-63. 